\title{
Ultrasound compared with clinical examination in infantile hypertrophic pyloric stenosis
}

\author{
P Godbole, A Sprigg, J A S Dickson, P C Lin
}

\begin{abstract}
Objectives-To assess the accuracy of clinical examination as compared with ultrasound imaging in the diagnosis of infantile hypertrophic pyloric stenosis. Duration of hospital stay, time between admission and surgery, and financial implications were also considered.

Design-A prospective study of patients referred to the surgical team with a possible diagnosis of pyloric stenosis from May 1993 to January 1995.
\end{abstract}

Setting-Neonatal and paediatric surgical wards and imaging department of a paediatric teaching hospital.

Subjects-116 patients referred to the surgical team with a possible diagnosis of pyloric stenosis.

Results -75 patients in this study had pyloric stenosis (64.6\%). Clinical examination had a sensitivity of $72 \%$, specificity of $97 \%$, with a positive and negative predictive value of $98 \%$ and $61 \%$ respectively. There were 16 diagnostic errors (one false positive and 15 false negative). Ultrasound imaging had a sensitivity of $97 \%$, specificity of $100 \%$, with a positive and negative predictive value of $100 \%$ and $\mathbf{9 8 \%}$ respectively. There was one diagnostic error (one false negative). Eight patients required repeat scans for confirmation of the diagnosis. On review of the initial scans in these patients, seven were noted to have inaccurate measurements due to poor technique. The average time between repeated scans was $\mathbf{2 8 . 2}$ hours. Ultrasound imaging cost $£ 13.90$ per scan and initiated a change in management only in the clinically false negative group at a cost of $£ 52$ per patient. The average duration of hospital stay was 3.1 days and the mean time between admission and surgery was 19.2 hours. The total cost for treatment of a patient with pyloric stenosis was $€ 1602$.

Conclusion-Ultrasound imaging should be reserved for those cases where clinical examination is negative and should be carried out by sonographers who see enough cases to maintain their expertise. (Arch Dis Child 1996;75:335-337)

Keywords: infantile hypertrophic pyloric stenosis, ultrasound, clinical examination.

Infantile hypertrophic pyloric stenosis occurs in about $3 / 1000$ live births, ${ }^{1}$ the diagnosis being made primarily by palpation of the hypertrophied pylorus. ${ }^{2}$ In 1977 Teele and Smith $^{3}$ described the use of ultrasound in the diagnosis, a technique which has rapidly gained popularity and is currently proposed to be the diagnostic procedure of choice. ${ }^{4-7}$ The pyloric muscle index was described in $1988^{8}$ and was found to be more reliable than previous measurement criteria for the diagnosis on ultrasound scan. ${ }^{4}$ Increasing reliance on radiological imaging and reduction in doctors' skills in clinically diagnosing pyloric stenosis have been demonstrated. ${ }^{9}$ This trend towards routine ultrasound imaging rather than clinical examination has been questioned. ${ }^{4}$ Restricting the use of imaging to patients with equivocal or indeterminate diagnostic features has been recommended. ${ }^{24}{ }^{4}$

This study was planned to assess the efficacy of ultrasound compared to clinical examination in the diagnosis of infantile hypertrophic pyloric stenosis with respect to diagnostic reliability, possible delay in surgical intervention, duration of hospital stay, and cost effectiveness.

\section{Methods}

A prospective study was undertaken of patients referred to the surgical team with a possible diagnosis of pyloric stenosis from May 1993 to January 1995. All patients who underwent both clinical examination and ultrasound imaging were included in the study. Palpation of the hypertrophied pylorus by a single experienced clinician was considered as a positive clinical examination. After the clinical examination, ultrasound imaging was performed, the radiologist being unaware of the outcome of the clinical examination. The patient was not starved or given any special preparation before the scan. The pyloric muscle index ${ }^{8}$ was used as the diagnostic criterion on scan and an index of $>0.46$ was considered to be positive for pyloric stenosis. The duration between admission and surgery and duration of hospital stay was calculated from the clinical and operative notes. The cost of ultrasound imaging was calculated.

To make this study as close as possible to our usual practice, we tried to ensure that there was no particular bias towards which clinician or radiologist performed the examination. The examinations were carried out by middle grade staff with consultant supervision. In the majority of cases, ultrasound scans were performed within normal sessions, the remainder by the on-call radiologist out of hours. 


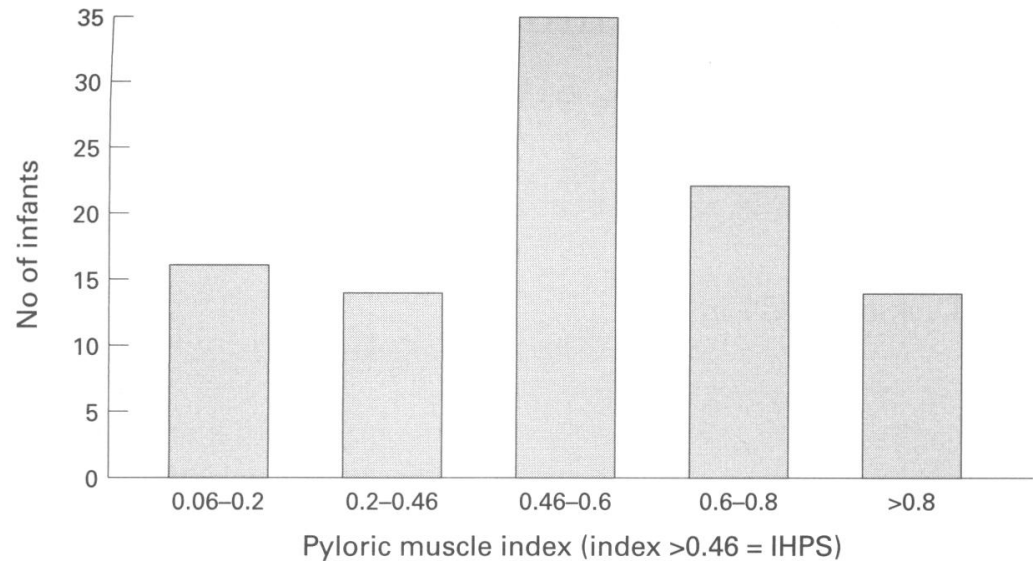

Figure 1 Distribution of pyloric muscle indices.

\section{Results}

One hundred and sixteen patients were included in the study. Seventy five of these had pyloric stenosis and in all cases this was confirmed at operation $(64.6 \%)$. The male to female ratio of patients with pyloric stenosis was 5.7:1. The mean age at presentation was 38.3 days with a range of 12 to 140 days. The mean weight at presentation was $4.02 \mathrm{~kg}$ with a range of 2.43 to $7.10 \mathrm{~kg}$. The mean duration of hospital stay was 3.1 days and the mean duration between admission and surgical intervention was 19.2 hours. Variables to be accounted for included availability of theatre space, biochemical imbalance, state of hydration of the patient, availability of senior anaesthetists, and time waiting for the scan to be performed.

\section{CLINICAL EXAMINATION}

One hundred and twenty six examinations were carried out on the 116 patients. Of 75 patients with pyloric stenosis, clinical examination was positive in $60 / 75(80 \%)$. There was one false positive examination, disproven on ultrasound imaging, surgery being avoided. In the remaining 15 patients, pyloric stenosis could not be clinically detected (20\%). Clinical examination was in agreement with the outcome in 40/41 patients who did not have pyloric stenosis, defined by resolution of vomiting. Clinical examination therefore had a sensitivity of $72 \%$, specificity of $97 \%$, positive predictive value of $98 \%$, and a negative predictive value of $61 \%$.

\section{ULTRASOUND EXAMINATION}

One hundred and twenty seven scans were performed on the 116 patients. Of the 75 patients with pyloric stenosis, ultrasound examination was confirmatory in $74 / 75(97 \%)$, eight on repeat scan. There was one false negative scan in an infant proven to have pyloric stenosis at surgery. No false positive scans were noted in this study. The remaining scans were normal, no patients having pyloric stenosis on follow up.

The indication for a repeat scan was persistence of vomiting and a high clinical suspicion of pyloric stenosis, despite a negative clinical examination. In a retrospective review of the initial scans of the eight patients proven to have pyloric stenosis at surgery, it was noted that in
Table 1 Annual cost of ultrasound scan-1994

\begin{tabular}{ll}
\hline Item & $£$ \\
\hline Machine - $£ 48000$ (over 5 years) & 9600 \\
Capital costs (6\%) & 2880 \\
Maintenance & 5600 \\
Sonographer & 19400 \\
Radiologist (3 sessions) & 13500 \\
Films/chemical/gel & 950 \\
Subtotal & 51930.00 \\
$+10 \%$ administative costs & 57123.00 \\
Cost per scan & 13.90 \\
Cost of 127 scans & 1765.30 \\
\hline
\end{tabular}

seven cases the initial scans were considered negative for pyloric stenosis because of inaccurate measurements due to poor technique. All these patients had had a negative clinical examination. The average delay between repeating the scans was 28.2 hours (range 6 to 72 hours). The remaining patient was noted to have accurate measurements on all the scans. It was only in this patient that the pyloric hypertrophy was noted to increase over a period of 10 days.

There was a single false negative ultrasound scan in this study, clinical examination being positive for pyloric stenosis, which was confirmed at operation. On retrospective review of the images, this was noted to be a borderline scan, but not falling within the diagnostic range according to the pyloric muscle index. There were no false positive scans in this study. Ultrasound imaging therefore had a sensitivity of $97 \%$, specificity of $100 \%$, positive predictive value of $100 \%$, and a negative predictive value of $98 \%$.

The distribution of pyloric muscle indices is shown in fig 1 and the method of calculating the cost of an ultrasound scan $(£ 13.90)$ detailed in table 1 . The total imaging costs for 127 scans were $£ 1765$.

\section{Discussion}

Ultrasound has been shown to be reliable and cost effective in the diagnosis of pyloric stenosis in comparison to clinical examination. There was no appreciable delay in surgical intervention or duration of hospital stay because of the wait for ultrasound imaging in the majority of patients who proved to have pyloric stenosis at operation. There were, however, eight patients who had a negative clinical examination in whom there was delay due to the need to repeat the ultrasound scan and the clinical examination before confirming the diagnosis of pyloric stenosis. The decision to repeat the scans was primarily a clinical one, based on persistence of vomiting. The initial scan in these eight cases was negative according to the pyloric muscle index $(<0: 46){ }^{8}$

Ultrasound is non-invasive, does not use ionising radiation, and reduces the need to perform repeated clinical examinations. However, it is an operator dependent procedure and can occasionally be difficult, even for the experienced radiologist. Inaccuracy in linear measurements may mean the difference between a positive and negative result. We ensured that clinical and ultrasound examination were performed by the usual grade of medical staff to avoid bias. Extrapolation of our results 
obtained in a paediatric teaching hospital unit may not be applicable to other centres. Diagnostic facilities including the necessary experienced personnel may not always be available in hospitals other than regional paediatric centres, and restricted access to ultrasound may cause diagnostic delay. Previous reports have shown that the request rate for radiological examinations, either ultrasound or barium meal examination, is between $6 \%$ and $23 \%$ in district general hospitals. ${ }^{11}$ This could be a limiting factor in gaining experience in radiological diagnosis of pyloric stenosis, hence the need for clinical examination as the first step in the diagnosis of pyloric stenosis. No barium studies were performed in this series.

The cost of a standard cot per day for a patient with pyloric stenosis in our hospital during this study was $£ 304$. The cost of operative treatment per patient was $£, 660$ for theatre costs alone. Hence the cost of treatment of a patient with pyloric stenosis with an average hospital stay of 3.1 days was $£ 1602$. The delay in the patients with pyloric stenosis who had to wait for a repeat scan therefore incurred an extra cost of $£ 357$ per patient.

In the clinically negative group $(n=56)$, including both patients without pyloric stenosis and the false negative group, ultrasound initiated a change in management in 15 patients $(26 \%)$. This incurred a cost of $£ 778$ to diagnose pyloric stenosis in 15 patients, that is, $£ 52$ per patient with pyloric stenosis. Referring to the previous figure, we feel this is costeffective, since it allows for earlier diagnosis and decreased hospital stay.

If all babies with possible pyloric stenosis had an ultrasound scan, there could be a waste of resources and this could lead to a loss of clinical skills in diagnosing pyloric stenosis. Similarly, if too few ultrasound scans are carried out, the experience of ultrasonographers may not be sufficient to maintain the level of expertise required in diagnosing pyloric stenosis. If only the clinically negative patients had ultrasound scan, in this study only 15 of 56 patients would have had a positive scan over a 17 month period. This might be insufficient to maintain sonographic expertise. Hence some cases of pyloric stenosis diagnosed clinically may need to be scanned from time to time. Although this has a cost implication, it would not be detrimental to the babies as ultrasound is non-invasive and safe.

In this study, eight out of 75 cases of pyloric stenosis had an initially false negative ultrasound, seven of which were thought retrospectively to be due to inaccurate measurements. The distribution of pyloric muscle indices conformed to a normal distribution curve, with no clear demarcation at an index of 0.46 between positive and negative groups as was reported in a previous study from this hospital. ${ }^{8}$ This could reflect the fact that reliance on the pyloric muscle index is too rigid and as the line of demarcation this figure may need to be reviewed. Furthermore many experienced ultrasound operators look upon the diagnosis of pyloric stenosis as a dynamic examination, relying less on measurements and more on direct visualisation of the pylorus and its behaviour, and claim that the false negative and false positive rate is virtually zero.

The criteria for repeat scans were persistence of vomiting and a strong index of suspicion of pyloric stenosis, despite a negative clinical or ultrasound examination. This selected the patients needing a repeat scan from the others in whom the vomiting resolved spontaneously.

\section{CONCLUSION}

We conclude that ultrasound imaging need not be carried out as a first line investigation for the diagnosis of pyloric stenosis, especially where it is not being carried out on a routine basis. Any radiologists or sonographers carrying out ultrasound for possible pyloric stenosis should see enough cases to maintain their expertise. Patients in whom the hypertrophied pylorus can be reliably palpated by an experienced clinician need not undergo ultrasound imaging. The use of ultrasound imaging should be reserved for those cases where clinical examination is negative. In this study, ultrasound prevented a negative laparotomy in the single clinically false positive case. This would seem to go against our previous recommendation. There were no false positive ultrasound examinations in this study. However, these are known to occur and in the long term would result in negative laparotomies. In this study, the clinically false positive case accounted for $0.8 \%$ of the total examinations. In a previously reported series, false positive and false negative ultrasound accounted for $1 / 147(0.7 \%)$ and $21 / 147(1.3 \%)$ of the total ultrasound examinations. ${ }^{10}$ We would therefore expect the negative laparotomy rate to be comparable in the long term, irrespective of the mode of diagnosis. The use of two separate clinical opinions can help in reducing diagnostic errors, with ultrasound imaging reserved for cases where there is a difference of opinion.

1 Anonymous. Incidence of infantile hypertrophic pyloric stenosis. Lancet 1984; i:888-9.

2 Forman HP, Leonidas JC, Kronfeld GD. A rational approach to the diagnosis of hypertrophic pyloric stenosis: do the results match the claim? $\mathscr{F}$ Pediatr Surg 1990;25:262-

3 Teele RL, Smith EH. Ultrasound in the diagnosis of idiopathic hypertrophic pyloric stenosis. N Engl $\mathcal{f} \mathrm{Med}$ 1977;296:1149-50.

4 Anonymous. Is ultrasound really necessary for the diagnosis of hypertrophic pyloric stenosis? Lancet 1988;i:1146.

5 Khamapirad T, Athey PA. Ultrasound diagnosis of hyperKhamapirad T, Athey PA. Ultrasound diagnosis
trophic pyloric stenosis. $\mathcal{f}$ Pediatr 1983;102:23-6.

6 Wilson DA, Vanhoutte JJ. The reliable sonographic diagnosis of hypertrophic pyloric stenosis. $f$ Clin Ultrasound 1984; 12:201-4.

7 Haller JO, Cohen HL. Hypertrophic pyloric stenosis; diagnosis using US. Radiology 1986;161:335-9.

8 Carver RA, Okorie M, Steiner GM, Dickson JAS. Infantile hypertrophic pyloric stenosis-diagnosis from the pyloric muscle index. Clin Radiol 1988;38:625-7.

9 Macdessi J, Oates RK. Clinical diagnosis of pyloric stenosis: a declining art. BMF 1993;306:553-5.

10 Neilson D, Hollman AS. The ultrasonic diagnosis of infantile hypertrophic pyloric stenosis; technique and accuracy. Clin Radiol 1994;49:246-7.

11 Erikson CA, Anders CJ. Audit of results of operations for infantile pyloric stenosis in a district general hospital. Arch Dis Child 1991;66:130-3. 